\title{
Futuros Docentes y Futuros Investigadores se Expresan sobre el Modelado en Física
}

\author{
Stella Maris Islas \\ Departamento de Formación Docente, Facultad de Ciencias Exactas \\ Universidad Nacional del Centro de la Provincia de Buenos Aires \\ Campus Universitario, Tandil (7000), Argentina \\ sislas@exa.unicen.edu.ar \\ Marta A. Pesa \\ Departamento de Física, Facultad de Ciencias Exactas y Tecnología \\ Universidad Nacional de Tucumán, Av. Independencia 1800 - Tucumán (4000), Argentina \\ mpesa@herrera.unt.edu.ar
}

Recebido em 6 de Julho de 2001. Aceito em 1 de Outubro 2001.

\begin{abstract}
Se presenta una investigación exploratoria sobre las concepciones de modelo científico sustentadas por estudiantes universitarios de Licenciatura en Física y de Profesorado en Física. A partir de entrevistas individuales semi-estructuradas se indagaron algunas ideas clave acerca del modelado y del empleo de modelos. Los resultados muestran a los futuros licenciados con una postura frente al tema que es acorde con las consensuadas en las epistemologías actuales, y se registran evidencias de su experiencia en el manejo de modelos. Ello no ocurre con los futuros docentes, quienes presentan ms acentuadamente las dificultades que, sobre este tema, se han detectado en otras investigaciones con estudiantes.
\end{abstract}

An exploratory research on scientific model's conceptions sustained by university students of Physics and Physics Teaching is presented. Starting from individual semi- structured interviews, some key ideas were investigated about modeling and the use of models. The results show that future physicists adopt a posture in agreement with the consensual ones in the current epistemologies. Also, evidences of their experience in the handling of models are registered. Among the future teachers the situation is different. They present difficulties similar to those that have been detected in other researches with students.

\section{Introducción}

Se presentan los resultados de una investigación exploratoria sobre modelado en Física, realizada en una población estudiantil universitaria. Enmarcada en un estudio más amplio (en el cual se indagó sobre este tema también en docentes y en investigadores en actividad), esta investigación muestra diferencias en la conceptualización de modelo científico entre estudiantes avanzados de Profesorado en Física y de Licenciatura en Física. Estos últimos ya están iniciados en tareas de investigación y manifiestan conceptualizaciones de modelo más afines a las consensuadas en las comunidades de investigadores (en Física, y en Educación Científica) que son, a su vez, consistentes con posturas epistemológicas contemporáneas (tales como las de Laudan, 1986; Bunge, 1985; Snyder, 2000; Bachelard, 1991).

Consideramos interesante estudiar esta prob- lemática, teniendo en cuenta que la relevancia del modelado en la construcción del conocimiento científico erige a esta estrategia como uno de los contenidos procedimentales que deberían tratarse explícitamente en la educación científica en todos los niveles y más aún entre los futuros docentes, ya que la competencia y la formación epistemológica en este modo de conocer los colocaría en mejores condiciones de abordarlo apropiadamente en su futura actividad docente. La investigación educativa en Física ha mostrado que tal competencia es necesaria para que ciertas dificultades de aprendizaje escolar relacionadas con el empleo de modelos puedan paulatinamente superarse (Ej.: Meyling, 1997; Ryder et al., 1999)

El propósito de este trabajo es ofrecer elementos que sean útiles para avanzar en el estudio de esta problemática. 


\section{Los modelos en la ciencia}

Desde distintas perspectivas epistemológicas (Bunge, 1985; Toulmin, 1977; Laudan, 1986) se reconoce el carácter de mediadores que tienen los marcos teóricos cuando se aborda el estudio de los fenómenos físicos. El científico, dentro de un marco conceptual, asigna los primeros significados al hecho que observa, lo cual le permite acotar los espacios de búsqueda de explicaciones, y reducir la extensión del universo de hipótesis que podría formular. Asimismo, se hace fecundo el planteamiento de problemas, ya que la observación orientada por hipótesis lleva a construir conexiones relevantes entre diversos hechos y estas conexiones posibilitan, a su vez, la resolución de los problemas (Bunge, 1985, pág. 418). Todos los problemas se plantean en el seno de un determinado contexto de indagación; y el mundo es siempre percibido "a través de las 'lentes' de alguna retícula conceptual”. (Laudan, 1986, pág. 43).

Desde esta visión, el científico no dispersa su atención hacia los detalles insignificantes, sino que se concentra en la esencia de lo que estudia. Bachelard (1991, pág. 261) ejemplifica con "El color de un proyectil no modifica sus propiedades balísticas" cuando alude a las estrategias de las cuales se vale el científico para acotar el conjunto de variables a considerar.

La simplificación practicada mediante la eliminación de variables no relevantes es una práctica que $M$. Bunge (1985) califica como una "regla de la construcción de teorías". De este modo, se tienen representaciones de los sistemas reales que este autor llama modelos ideales: un modelo no cubre la totalidad de los aspectos del sistema real que representa, sino sólo aquellos que tienen relevancia a la luz de la teoría que hace referencia a ese sistema. El autor seala la potencialidad de las construcciones teóricas para trascender los hechos, y ofrecer explicaciones cuya riqueza deriva, precisamente, del hecho de haber tomado los aspectos que constituyen las variables relevantes de los variados fenómenos a los cuales aluden, y de la delimitación de su campo de aplicación.

Frente a este carácter genérico de los modelos abstractos (Nersessian, 1995) podemos considerar los modelos más específicos (Snyder, 2000) que son aquellos que se disean para abordar el estudio de un fenómeno en particular. La selección de variables, además de estar orientada por la teoría, está marcada por las condiciones particulares del problema a resolver, y por las herramientas (formales y experimentales) con las que el científico aborda la resolución de ese problema.

Es posible, entonces, representar el fenómeno en estudio bajo diversos formatos, y se escoge uno de ellos por ser el que se muestra más apto para la investigación a realizar; a veces, el avance de la tarea de investigación demanda correcciones al modelo en uso. En este trabajo aludimos a la posibilidad de elaborar distintos modelos para una misma situación real con la expresión flexibil- idad del modelado.

Tanto esta "flexibilidad" como la "simplificación" que forma parte del proceso de modelización, se relacionan con el tipo de correspondencia que existe entre un modelo y su correlato empírico: tal correspondencia no es puntual, sino que es estructural (esto es, de sistema a sistema, que implica una relación de analogía). De ello deriva la existencia de modelos alternativos para un cierto sector de la realidad (los "múltiples modelos" de los que hablan Grosslight et al., 1991). Un sistema real puede representarse de diferentes maneras, cada una de ellas construida en función de las herramientas (conceptuales y experimentales) disponibles y dependiendo de los propósitos particulares de la investigación (Islas-Pesa, 2000 a; Snyder, 2000; Harrison- Treagust, 2000).

Dado que la modelización no tiene el propósito de copiar a la realidad, existe siempre una brecha entre modelo y realidad, que se salva mediante la contrastación experimental (Cudmani et al, 1991). En este proceso juegan un rol fundamental la convalidación de resultados y la contrastación experimental, que permiten delimitar los campos de validez del modelo y de las conclusiones obtenidas mediante su empleo. Así, la evaluación de los errores experimentales "constituye un criterio cuantitativo fundamental para controlar si es posible o no 'modelizar' de tal o cual manera una situación concreta y predecir comportamientos fácticos" (op. cit., pág. 54).

Cada una de estas modelizaciones concretas que elabora un científico da lugar a lo que J. Gilbert et al (1998) llaman "modelo expresado", que es sometido a la consideración de la comunidad en la cual está inserto. Si ésta lo aprueba, se convierte a este modelo en un "modelo consensuado", que pasa a formar parte del acervo de saberes científicos. La idea de estas sucesivas transformaciones de los modelos es considerada también en Gobert-Buckley (2000), Islas-Pesa (2000 a, b), Justi-Gilbert (2000), Harrison-Treagust (2000).

En los trabajos recién referidos se relaciona, además, al modelo consensuado con los "modelos de enseñanza": "Un modelo de enseñanza es un modelo expresado especialmente construido, que usan los docentes como ayuda para la comprensión de un cierto modelo consensuado" (J. Gilbert et al, 1998).

\section{La investigación educativa sobre modelos científicos}

La transformación de un modelo científico consensuado en un modelo de enseñanza, asícomo el empleo de estos últimos en las aulas y los resultados de aprendizaje al respecto, son objeto de investigaciones cuyos marcos teóricos son consistentes con el que acabamos de sintetizar. Se indaga sobre las concepciones de modelo en aprendices de Ciencias de diferentes edades y características (ejemplos: S. Gilbert, 1991; Grosslight et al, 1991; Meyling, 1997; Harrison-Treagust, 1996, 2000; 
Ryder et al. , 1999; Snyder, 2000). Entre los resultados de investigación sobre estudiantes son especialmente interesantes los siguientes:

- Los estudiantes conciben, frecuentemente, al modelo como una "copia" de la realidad; por ello, no reconocen la "flexibilidad" del modelado a la cual hemos aludido en el apartado anterior.

- Los estudiantes tienen dificultades para distinguir entre un modelo y la realidad que él representa; esto es más notable cuando esa porción de la realidad está conformada por entidades no accesibles a la percepción directa.

- Las expresiones matemáticas relacionadas con un modelo suelen ser manejadas por los estudiantes sin que sean reconocidas las entidades físicas a las cuales corresponden.

- El rol del científico en la construcción de modelos, asícomo la importancia de la validación comunitaria de ellos no son asuntos reflexionados habitualmente por los estudiantes.

- Tampoco es frecuente que los estudiantes reconozcan con facilidad el carácter sistémico de la relación modelo-teoría. Tienden a relacionar la validez de un modelo más fuertemente con la contrastación empírica que con la validez teórica.

Progresos en la comprensión del modelado han sido logrados mediante la implementación de estrategias didácticas destinadas a tal fin, tal como lo prueban los estudios de I. Halloun (1996), Meyling (1997). Acordamos con Aiello y Sperandeo (2000) cuando afirman que "la implementación de nuevos enfoques en la educación en Física exige la implementación de nuevos métodos en la formación docente", puesto que los profesores necesitan un conocimiento profundo de la naturaleza de los modelos físicos y de su funcionamiento en el desarrollo de la disciplina tanto como una toma de conciencia de los modelos espontáneos de los estudiantes en las diferentes áreas de contenido. Sobre la base de este conocimiento los docentes podrían proporcionar a sus alumnos "el tiempo y la ayuda" que necesitan para comprender el carácter representacional de los modelos (Harrison-Treagust, 2000). El trabajo de Aiello- Sperandeo (2000) proporciona un ejemplo de investigación exploratoria en la que se prepara especialmente a futuros docentes para desenvolverse en cuestiones de modelado.

A partir de los resultados de investigación que acabamos de resear pueden ser formulados, para nuestra población, los interrogantes que siguen:

¿cómo interpretan estos estudiantes a la relación entre un modelo y el sistema real que él representa? ¿aparece la noción de modelo como "copia” de la realidad?

¿reconocen la "flexibilidad" del modelado? ¿tienen dificultades para reconocer los referentes fácticos de las expresiones matemáticas involucradas en un modelo?

¿qué criterios proponen para establecer la validez de un modelo?

¿cuáles son sus ideas respecto de los propósitos que persigue la elaboración de modelos en física?

¿pueden señalarse diferenciaciones en sus posturas que sean atribuibles al tipo de carrera (profesorado/licenciatura) que están finalizando?

los estudiantes de profesorado ¿están encaminados hacia una competencia para abordar los tópicos de modelado con sus futuros alumnos?

\section{Metodología}

La literatura de investigación en educación en Física brinda informaciones acerca del aprendizaje del modelado en estudiantes que -en la mayoría de los casosno son universitarios; por ello consideramos que el esquema exploratorio (Samaja, 1993, pág. 271) es el más apropiado para registrar información sobre este tema en estudiantes universitarios, que dé cuenta de unos primeros indicadores susceptibles de ser desarrollados en el futuro y que sea fecunda en la formulación de nuevas hipótesis. Nuestra intención no es, entonces, proponer una generalización de estos resultados, sino aportar elementos para proseguir el estudio del tema.

Habiendo decidido explorar en profundidad las concepciones de modelo en nuestros estudiantes, adoptamos una metodología cualitativa, para realizar entrevistas semi-estructuradas individuales. Usando un protocolo que contiene preguntas abiertas (Ver Anexo 1) se promovió con cada estudiante la instauración de un diálogo fluido, en el cual ellos pudieran agregar comentarios que fueran valiosos para configurar una caracterización adecuada de sus concepciones de modelo y del rol del modelo en la construcción del conocimiento científico.

Se trabajó sobre la población conformada por estudiantes avanzados (4⿳⺈ año en adelante) de las carreras de Licenciatura ${ }^{1}$ en Física y Profesorado ${ }^{2}$ en Física de la Facultad de Ciencias Exactas de la Universidad Nacional del Centro de la Provincia de Buenos Aires. La muestra (de tamaño 6) reviste el carácter de "sustantiva" (Samaja, 1993) por haber sido elegida con criterio no aleatorio, fundamentando su analogía con la población en un conjunto de datos previamente conocidos acerca de la misma. Entre tales antecedentes, cabe citar como ejemplos: plan de estudios que ha cursado cada estudiante, grado de avance en la carrera, aprobación de asignaturas de fuerte componente profesional (Laboratorio en estudiantes de Licenciatura/

\footnotetext{
${ }^{1}$ En las universidades argentinas, la Licenciatura es una carrera de seis años, que finaliza con la aprobación de una Tesis (trabajo de investigación en la especialidad correspondiente)

${ }^{2}$ En las universidades argentinas, el Profesorado es una carrera de cuatro años.
} 
Práctica de la enseñanza en los de Profesorado), actividades de investigación (Tesis de grado en los de Licenciatura/ vinculación con investigadores en educación científica en los de Profesorado). Desde el conocimiento de la variabilidad de estos antecedentes en la población estudiantil, se conformó una muestra cuya estructura responde a la de la población (Firestone, 1993).

Los datos emergentes de las transcripciones literales de las entrevistas fueron procesados mediante la construcción de un sistema de categorías (Dey, 1993), que permitió preservar la contextualización de los segmentos de discurso, debido a que el empleo del sistema demanda una inspección recurrente de datos y categorías. De este modo, los registros quedan sistematizados en las categorías que se detallan en la siguiente sección.

\section{Resultados}

En la primera parte de esta sección, se detallan los resultados obtenidos para cada categoría, incluyendo en algunos casos la transcripción de segmentos de discurso. Cabe aclarar que por razones de espacio, esta presentación no detalla la totalidad de los resultados registrados con estos estudiantes. Se expone sobre el grupo de categorías que atienden a una de las variables en estudio (concepción de modelo científico); en tanto que solamente referimos una de las categorías de la otra variable (experiencias personales en el manejo de modelos). Es la categoría que compila la información obtenida al analizar las respuestas de los entrevistados acerca de los modelos de uso más frecuente en las clases universitarias de las cuales ellos participan.

A los efectos de facilitar la lectura, es pertinente aclarar la notación usada aquí:

F.D. = futuro docente (estudiante de Profesorado en Física)

F.I. = futuro investigador (estudiante de Licenciatura en Física)

Luego se presentan cuadros que resumen las manifestaciones de los estudiantes de cada grupo, con el propósito de facilitar la posterior lectura de las conclusiones en las que se comparan ambos grupos.

\section{Categoría 1: El modelo, y su relación con la re- alidad}

Aparecen escasas referencias al carácter analógico de esta relación. Sólo una de las F.D. se refiere explícitamente al modelo como representación análoga de la realidad, pero se trata de una estudiante que ha cursado asignaturas en las que estudió el tema de los Modelos mentales, ha cursado Epistemología de la Física, y ha tenido acceso a literatura de investigación educativa. Diferente es la formación de los demás estudiantes, quienes han cursado el Profesorado con un Plan de Estudios anterior (que no incluía estos temas) o bien, son F.I. en cuya carrera no se ha abordado curricularmente esta temática.

Con diferentes grados de acentuación aparece la noción de modelo como representación simplificada de la realidad. En el comienzo de la entrevista a uno de los F.I. se registra: "Probablemente, la idea es tratar de simplificar las cosas, para ver cuál es el fenómeno dominante. Por ahí, lo que pasa en Física es eso: que están todos los fenómenos juntos, un montón de fenómenos juntos, pero todos tienen... este... diferentes órdenes de magnitud. Entonces, la forma de estudiar de la Física es tratar de sacar, digamos, qué es lo relevante. Porque, por un lado, va a ser lo más simple, va a ser el modelo más simple, seguramente; y por otro lado, va a ser lo que dé las características generales del problema." Se presenta aquíal recorte simplificatorio asociado con la posibilidad de tener una visión general del problema. Este estudiante es discípulo de un científico que trabaja en modelado y su apreciación de la visión general de un problema, difiere de la de otro F.I. que trabaja en Física experimental, quien manifiesta: “... en la parte experimental tenés que tener en cuenta todo, y en el papel, bueno, podés suponer un montón de cosas que te facilitan los cálculos (...) Por eso, me atrapa más a mila parte experimental que la teórica. O sea, el estar pendiente constantemente de todas las variables que están dando vueltas, de si tenés la temperatura ambiente que bajó, que si la humedad, que si esto, que si aquello..."

El otro F.I. coloca a la palabra "simplificación" como la primera que viene a su mente al pensar en modelo y retoma la noción en los últimos párrafos de la entrevista, cuando está hablando de la relación modelorealidad.

Menos explícitos son los discursos de los F.D., ya que se limitan a mencionar a los recortes simplificatorios, pero sin resaltar el rol de la simplificación en la construcción de modelos.

Sólo se encuentra un episodio en el que se alude al modelo dentro de un camino de aproximaciones sucesivas al hecho real: "Para mí, el modelo, en la parte experimental, es como la primera aproximación a la experiencia. Yo estoy seguro de que, por ejemplo, en ningún modelo debe estar contemplado que para no tener el problema antena tenés que retorcer el cable. O sea, a ese tipo de cosas, me refiero. En la primera aproximación, te va a acercar, o sea, vas a tratar de reproducir todas las condiciones que te impone el modelo, y bueno, vas a decir: vamos a ver, de acá en adelante, qué es lo que me pasa. O sea, eso es más o menos, el concepto que yo tengo de modelo". En otro momento del diálogo, este F.I. coincide con dos de los F.D. en afirmar que es deseable que el modelo esté lo más cerca posible del hecho que representa. Pero más adelante, "pensando en voz alta", se retracta de lo antedicho, y concluye que sería demasiado complicado manejar un modelo cuyas ecuaciones intenten 
tener en cuenta todas las variables. Esta consideración pragmática está presente, también, en lo que afirman sus dos compañeros F.I.

Otro de los F.I. relaciona a esta consideración con las condiciones impuestas a la realización de experimentos: "Entonces, por ahi, en esto de modelo y realidad, hay que ver qué realidad uno quiere describir, qué pretende. Si uno acomoda las cosas como para que la realidad que está viendo, o la que está midiendo, porque ahíestá la cosa: no es que el modelo tanto se acerque a la realidad, son las dos cosas. Uno los experimentos los prepara para ver lo que realmente quiere estudiar."; unas líneas más adelante, este estudiante comenta que atiende sólo a aquellos aspectos de un fenómeno que, en función de los presupuestos teóricos involucrados en el modelado, son los que interesan para un determinado experimento. De este modo, este F.I. está destacando que el modelo es la construcción teórica que orienta el recorte de variables que se practica al proyectar los trabajos de laboratorio y al hacer los registros en el curso del experimento. (Estas consideraciones son consistentes con las sealadas en el Marco Teórico de este trabajo).

Entre los F.I. entrevistados, se advierte una tendencia a vincular el modelo con la realización de algún experimento para el cual el modelo es útil. Citan modelos consensuados (ej.: los utilizados para la configuración del átomo, los modelos de fluidos ideales, etc.), pero se explayan más en la consideración de modelos especialmente diseados para un experimento, y destacan que el recorte de variables está orientado por los propósitos de la situación problemática a resolver. Sus discursos quedan focalizados en los modelos que utilizan para resolver los problemas que abordan en su investigación, pero esta restricción en la temática de los diálogos es atribuible al interrogatorio que guía las entrevistas, el cual fue elaborado a partir del recorte del problema que estudia esta investigación.

\section{Categoría 2: Validez de los modelos}

La referencia a las actividades de experimentación es el punto de partida en la consideración de los límites de validez de cada modelo en las respuestas de los F.I., expresando el criterio para validar un modelo en términos de condiciones iniciales y de contorno, de discriminación de variables relevantes y no relevantes, y del marco impuesto por las simplificaciones practicadas.

En tanto que, de los tres F.D., sólo uno (quien estudió Epistemología) menciona el tópico de la validez, y lo propone desde el encuadre en una teoría: “... yo creo que el modelo tiene que estar de acuerdo, o tiene que... eh... todas las condiciones que se le pongan al modelo tienen que ser válidas dentro de esa teoría, para poder ser... para poder entrar dentro de la teoría. Y si hay cosas que no... que no entran dentro de esa teoría, no sé... entrará dentro de otra teoría, o habrá que formu- lar una nueva teoría para ese modelo, para ese nuevo modelo".

De acuerdo con lo asumido en el Marco Teórico de este trabajo, se esperaba que hubiera mayor profundidad de reflexiones acerca de la importancia de la convalidación y la contrastación experimental, la medición y la estimación de incertezas. Además, no se abundó en el añadido de preguntas en aquellos casos en que este tema fue citado por algunos de los estudiantes, para evitar el sesgo que puede generar el hecho de que la entrevista se desarrolla entre un profesor y un alumno de la misma Facultad.

La cita más explícita sobre este tema se encuentra en el discurso de una F.D. que es la única que menciona a la "teoría de los errores". Lo hace en el contexto de una valoración que considera que el modelo más elaborado es el que mejor "transcribe" a la realidad y que: "cuanto más uno trabaje ese modelo, y lo mejore, más cerca de la realidad va a estar. Pero yo creo que nunca va a ser la realidad, siempre va a haber un más-menos error".

En la misma entrevista se dan expresiones confusas acerca de la relación entre modelo y teoría, en tanto que en el caso de otra F.D. esta relación es presentada en la forma en la que se la toma en este trabajo, considerando al modelo como una construcción que se encuadra en una teoría.

Desde una postura similar, se da una mayor profundización en el tema de la relación entre modelo y teoría cuando uno de los F.I. relata explicaciones que ha recibido de su Director de Tesis: “... la diferencia, básicamente, según me explicó él, es que la gente que hace teoría, básicamente, se ocupa de ver... las teorías existentes cómo se pueden modificar. (...) Pero por ahiél se refería un poco... yo entiendo que a la gente... qué sé yo, volando mucho, no? como la gente que hace teoría de cuerdas, o que hace cosas como cosmología, o... un poco, gente que se dedica a hacer teoría, o trata de ver cómo se engancha la relatividad general con tal otra cosa. l decía que eso es hacer teoría. La gente que hace modelado es, en principio, gente que toma eso, toma eso como punto de partida, plantea esas ecuaciones, ecuaciones bien establecidas, como punto de partida y trata de aplicar esas ecuaciones a determinados fenómenos. Entonces elabora pequeos modelos, por asídecir, y eso es creo a lo que ellos llaman modelos. Por ejemplo, lo que yo hago, lo que hacen ellos, que es trabajar sobre láseres de semiconductores. En principio uno diría: bueno, un láser de semiconductores está basado en la Mecánica Cuántica, no se puede explicar de ninguna manera a través de la Física Clásica (...) toda la Fúsica del láser está basada en Mecánica Cuántica, seguramente. Ahora, estudiar el comportamiento del láser requiere un modelo, y un modelo que tiene que bajar de las ecuaciones."

Se registra aquíuna manifestación acorde con lo expresado en el Marco Teórico de este trabajo: el modelo 
representa a una porción de la realidad (Bunge, 1985).

\section{Categoría 3: Cómo trabajan los científicos con los modelos}

La pregunta acerca de los propósitos de la elaboración y del empleo de modelos genera respuestas que pueden agruparse en:

- Facilitar el estudio de un evento en el sentido de simplificar el estudio, volviéndolo accesible a las herramientas (formales y experimentales) disponibles. En este punto, coinciden las afirmaciones de tres estudiantes de ambas especialidades.

- "Reproducir" un fenómeno, sea bajo la forma de un experimento, de una formulación matemática, o de una simulación computacional.

Esta noción aparece en el discurso de uno de los F.I., quien lo reitera en varias oportunidades; una de ellas es: “...cuando escucho la palabra "modelo" pienso en algo que quiere reproducir un fenómeno. O sea, uno puede hablar de ... por ejemplo, ahora están tratando de simular modelos de por ejemplo volcanes, huracanes, cosas muy complicadas, y bueno, lo que tratan de hacer es estudiar el fenómeno y, con algunos parámetros que ellos conocen, tratan de reproducir el fenómeno justamente para, más que nada, poder por ahípredecirlo, o cosas por el estilo. O sea, que con ese modelo, tratan de tomar el caso natural, y matemáticamente poder reproducirlo; y después experimentalmente, no?".

- Para explicar y, además, predecir el comportamiento de la naturaleza. La capacidad predictiva de la modelización que fue recién mencionada como instancia relacionada con la "reproducción" de un fenómeno, es presentado por una F.D. en forma explícita.

- Para dirigir la observación y para posibilitar la interpretación de los datos son los propósitos que destaca otro de los F.I.

Opina que la recolección de datos sería estéril si no se cuenta con un modelo que permita interpretarlos y, además, en la misma recolección de datos se da un interjuego con el modelo: "a veces es un poco y un poco: uno mide algo, trata de obtener entonces cuál es el modelo y a partir de más o menos cómo es ese modelo, empezar a dirigir la observación en medir tal o cual cosa; ver cuán bien ajusta ese modelo".

Cuando se inicia el diálogo sobre el empleo de modelos alternativos para representar a un cierto hecho de la realidad en estudio, un F.I. expresa: " para estudiar diferentes procesos, se utilizan diferentes modelos. Un poco... bueno, esto es de la otra pregunta, de si se utilizan diferentes modelos para resolver una misma situación problemática. $Y$, sí, seguro. Incluso, a veces es útil, porque seguramente diferentes modelos encaran el problema desde diferentes... eh... diferentes...

$S:$ ¿miradas?

E: claro, que pueden ser complementarias. Este... por ahí, una cosa que es muy importante también, es que muchas veces es útil saber si determinado modelo es capaz de predecir determinada cosa. (...) Por ahí, uno ve experimentalmente tal cosa, y no sabe si tal modelo podrá reproducir, en principio, tal cosa o tal comportamiento que uno está viendo. Entonces, por ahí, el juego entre diferentes modelos: variar un término, sacar un término, es típico hacer ese tipo de cosas."

Desde esta perspectiva, fuertemente ligada a su trabajo en el laboratorio, el estudiante hace una afirmación de contenido muy afín a la que manifiesta, desde una perspectiva más general, una F.D.: la entrevistada acuerda con la posibilidad de que "dentro de la Ciencia" se acepten varios modelos para abordar el estudio de un mismo evento, y destaca la necesidad de optar por un único modelo para realizar una determinada tarea de investigación: “... trabajando, se tiene que aceptar uno como válido, y trabajar con ése, y saber que hay otros que en otro marco se utilizan de otra manera".

Estos dos estudiantes, aunque sus comentarios tienen diferente corte, se pronuncian de acuerdo en cuanto a la existencia de más de un modelo para una misma situación problemática. Esta postura es acorde con la adoptada en este trabajo, donde se asume que una misma teoría admite, para un hecho de la realidad, más de un modelo. El marco de validez de cada modelo, las herramientas (formales y experimentales) disponibles para utilizarlo, y los propósitos del investigador orientan la decisión de optar por uno u otro de los modelos que encuadran en la teoría.

La otra referencia en la que se encuentra un decidido acuerdo con el uso de más de un modelo incluye la comparación entre modelos de diferente nivel de complejidad, y contiene un enunciado consistente con otros registros: de los modelos más sencillos se derivan formulaciones cuya resolución es más simple que las que se relacionan con modelos más detallados. Un criterio para elegir entre diferentes modelos que representan a un mismo evento es, en opinión de estos F.I., el de reducir al mínimo la complejidad de las herramientas formales que demanda la resolución de cada problema.

En estrecha relación con las afirmaciones anteriormente comentadas están las que aluden a las posibilidades de modificaciones en los modelos que manejan los científicos. La evolución de un modelo se relaciona preferentemente con modificaciones puntuales practicadas sobre el modelo que se utiliza para resolver un problema concreto de investigación. Menos frecuente es la alusión a la evolución histórica de los modelos en el cuerpo de las construcciones científicas, pero este hecho debe interpretarse teniendo en cuenta la índole de las preguntas formuladas: ellas no apuntaban a disparar reflexiones epistemológicas acerca de la Ciencia como un todo, sino las concernientes a la actividad de científicos individuales. 


\section{Categoría 4: Empleo de modelos en las clases universitarias}

Si bien esta presentación no está centrada en la información recogida acerca de las experiencias personales de estos estudiantes con el modelado, consideramos importante incluir esta categoría, ya que ella muestra cómo ven estos jóvenes al manejo de modelos en las clases, asílos ejemplos concretos que ellos citan.

Los F.I. acuerdan en un modo más firme que los F.D. con la idea de que la construcción de modelos es una actividad que abarca a todos los temas que estudia la Física, considerando al modelado como una tarea central para la investigación, independientemente de cuál sea la porción de la realidad que se esté estudiando. Pero, por otro lado, ninguno de ellos ha tenido conciencia del uso de modelos hasta los últimos años de su carrera, esto es: durante los primeros años de su carrera de formación en la Universidad, no advertían que estaban usando modelos. Este hecho es apreciado de dos maneras diferentes: los F.I. están de acuerdo en que asísea, ya que opinan que las discusiones sobre modelos demandan una buena base de conocimiento previo en Física, y aptitudes para operar con elementos abstractos. Pero entre los F.D. aparece una valoración diferente: ellos creen que por desconocer la presencia de modelos, han tenido confusiones entre modelo y realidad, y que tales confusiones estarían entre las que pueden evitarse si el aprendiz es informado -desde el comienzo- de las relaciones modelo-realidad y modeloteoría.

En los testimonios recogidos, algunos de los ejemplos de temáticas en las cuales se emplean modelos aparecen como respuesta a una de las preguntas del protocolo de las entrevistas; pero la mayoría de las menciones se dan en el transcurso de los diálogos sobre diferentes tópicos.

Los ejemplos mencionados por los estudiantes admiten la siguiente tipología:

- Algunos de los ejemplos que dan los F.I. son los específicos de su tema de investigación, como el modelo de equilibrio termodinámico para un plasma irradiado con láser. También aportan algunos ejemplos no tan frecuentemente nombrados, como los elaborados para el estudio de fenómenos atmosféricos.

- Ambos grupos de estudiantes mencionan ejemplos que podrían denominarse "prototípicos", en el sentido de que comúnmente se alude a ellos usando el calificativo de "modelo", como el de los modelos atómicos, el modelo de un gas ideal, etc.

- En otros casos, la ejemplificación refiere a temáticas amplias: capítulos de la Física, tales como Cinemática, Dinámica; o bien a asignaturas de la carrera (Estructura de la materia, Física Cuántica, Astronomía).

Los resultados anteriormente referidos se presentan a continuación en el formato de cuadros que consignan las ideas clave de las manifestaciones de los estudiantes.
Las frases que figuran en letra cursiva contienen comentarios de las autoras.

\section{Análisis comparativo de los resultados}

El recorte de variables implicado en la construcción de un modelo científico aparece como la estrategia más fácilmente reconocida por estos estudiantes, en tanto que el carácter analógico de la relación entre modelo y realidad se muestra con menor evidencia. Los F.I. analizan el rol del recorte de variables en el diseño y en el uso de los modelos para la realización de estudios experimentales con mayor grado de detalle que los F.D., y la influencia de su práctica en la investigación puede notarse en los argumentos que esgrimen para fundamentar la necesidad de dejar de lado las variables no relevantes (complejidad del manejo de ecuaciones y de instrumental). Estas mismas son las razones por las cuales descartan la idea de que el modelo debería "copiar" a la realidad, intención que los F.D. atribuyen al modelado.

La validez de un modelo fundada en su encuadre en una teoría es únicamente mencionada por los dos estudiantes que tienen formación específica al respecto: una F.D. que ha cursado Epistemología y realiza colaboraciones con la investigación educativa en ciencias, y un F.I. cuyo director de Tesis es un científico que trabaja en modelado. Los demás F.D. no hacen esta referencia, y los F.I. detallan criterios relacionados con condiciones experimentales.

Las respuestas acerca de los propósitos que animan a un científico en el diseño y empleo de modelos pueden encuadrarse en concepciones consensuadas en la comunidad de investigadores. Las de F.D. giran en torno de cuestiones más generales que las de los F.I., quienes puntualizan diversas acciones concretas propias de la investigación científica.

En lo que concierne a la "flexibilidad" del modelado la diferencia entre los dos grupos se hace más notoria, ya que los F.I. se muestran totalmente seguros de la existencia de múltiples modelos posibles para resolver un determinado problema de investigación, mientras que los F.D. dudan al respecto, y conciben los cambios en un modelo en términos de "mejorar su ajuste a" (la teoría/ la realidad) sin tomar en consideración la funcionalidad del modelo como representación que permite estudiar científicamente la realidad, sea para explicar lo observable como para predecir el curso de los fenómenos.

Las experiencias personales de ambos grupos en el empleo de modelos en las clases universitarias tienen un atributo común: mientras fueron estudiantes "novatos" no tenían conciencia de la presencia del modelado. En el momento en que fueron entrevistados (es decir, sobre el final de su carrera de grado) sí reconocen haberlos empleado en las asignaturas de formación básica. Sus 
opiniones respecto de esta situación no son coincidentes:

los F.D. la califican como generadora de dificultades en

Categoria 1: Relación entre modelo y realidad

\begin{tabular}{||l|l|l|l||}
\hline & \multicolumn{1}{|c|}{ FUTUROS DOCENTES (F.D) } & \multicolumn{1}{c|}{ FUTUROS INVESTIGADORES (F.I.) } & \multicolumn{1}{c|}{ COMENTARIOS } \\
\hline $\begin{array}{l}\text { Relación } \\
\text { analógica entre el } \\
\text { modelo y la } \\
\text { realidad }\end{array}$ & $\begin{array}{l}\text { El modelo es una representación } \\
\text { análoga de un sistema real. (Lo dice } \\
\text { la única estudiante que ha cursado } \\
\text { Epistemologia). }\end{array}$ & $\begin{array}{l}\text { No se cita explícitamente; pero en varias } \\
\text { manifestaciones subyace esta noción. }\end{array}$ & $\begin{array}{l}\text { Se encuentran pocas referencias a esta } \\
\text { relación analógica. }\end{array}$ \\
\hline $\begin{array}{l}\text { El modelo como } \\
\text { representación } \\
\text { simplificada del } \\
\text { sistema real }\end{array}$ & $\begin{array}{l}\text { Los recortes simplificatorios se } \\
\text { emplean en el estudio de algunos } \\
\text { temas. (Pero no señalan el rol de la } \\
\text { simplificación en la construcción de } \\
\text { modelos). }\end{array}$ & $\begin{array}{l}\text { La simplificación permite acceder a una visión } \\
\text { general del problema de investigación. La palabra } \\
\text { "simplificación" fuertemente asociada a "modelo". } \\
\text { El modelo más sencillo como primera } \\
\text { aproximación al hecho en estudio. }\end{array}$ & $\begin{array}{l}\text { Ambos grupos coinciden en reconocer la } \\
\text { relevancia del recorte de variables. }\end{array}$ \\
\hline $\begin{array}{l}\text { Cantidad de } \\
\text { variables } \\
\text { incorporadas en } \\
\text { el modelo }\end{array}$ & $\begin{array}{l}\text { Es deseable que el modelo esté "lo } \\
\text { más cerca posible" del hecho real. }\end{array}$ & $\begin{array}{l}\text { Consideración pragmática (sería muy complicado } \\
\text { trabajar con más variables). Los experimentos se } \\
\text { diseñan para tomar en cuenta solamente las } \\
\text { variables que (según el modelo teórico) son } \\
\text { relevantes para resolver cada problema de } \\
\text { investigación. }\end{array}$ & $\begin{array}{l}\text { Las concepciones de los F. D. pueden } \\
\text { encuadrarse en la del modelo como "copia" } \\
\text { del sistema real. En tanto que las de los F. I. } \\
\text { presuponen una correspondencia estructural } \\
\text { (y no, puntual) entre el modelo y la realidad. }\end{array}$ \\
\hline
\end{tabular}

\section{Categoría 2: Validez de los modelos}

\begin{tabular}{|l|l|l|l|}
\hline & \multicolumn{1}{|c|}{ FUTUROS DOCENTES (F.D.) } & \multicolumn{1}{|c|}{ FUTUROS INVESTIGADORES (F.I.) } & \multicolumn{1}{c|}{ COMENTARIOS } \\
\hline $\begin{array}{l}\text { Límites de } \\
\text { validez }\end{array}$ & $\begin{array}{l}\text { La validez de un modelo se relaciona con } \\
\text { su encuadre en una teoría. (Única ocasión } \\
\text { en la cual se mencionan los límites de } \\
\text { validez). }\end{array}$ & $\begin{array}{l}\text { Los límites de validez dados en función de: a) las } \\
\text { condiciones del experimento; b) los órdenes de } \\
\text { magnitud de las variables relevantes; c) las } \\
\text { simplificaciones practicadas. Uno de estos estudiantes } \\
\text { detalla relación entre modelo y teoría. }\end{array}$ & $\begin{array}{l}\text { El control teórico es considerado por un } \\
\text { estudiante de cada grupo. } \\
\text { Los F.I. centran la validación de } \\
\text { modelos en el control experimental. }\end{array}$ \\
\hline $\begin{array}{l}\text { Medición, y } \\
\text { estimación } \\
\text { de } \\
\text { incertezas }\end{array}$ & $\begin{array}{l}\text { Una estudiante (que sostiene una } \\
\text { concepción de modelo como copia de la } \\
\text { realidad) cita a estas actividades. }\end{array}$ & $\begin{array}{l}\text { Acciones implícitamente consideradas en el control } \\
\text { experimental. }\end{array}$ & Las reflexiones no abundan en detalles. \\
\hline
\end{tabular}

Categoría 3: Cómo trabajan los científicos con los modelos

\begin{tabular}{|l|l|l|l||}
\hline \hline $\begin{array}{l}\text { Propósitos de la } \\
\text { elaboración y } \\
\text { del empleo de } \\
\text { modelos }\end{array}$ & $\begin{array}{l}\text { FUTUROS DOCENTES (F.D.) } \\
\text { Explicar y, además, predecir el } \\
\text { comportamiento de la naturaleza. }\end{array}$ & $\begin{array}{l}\text { FUTUROS INVESTIGADORES (F.I.) } \\
\text { "Reproducir" un fenómeno (experimento, } \\
\text { formulación matemática, simulación } \\
\text { computacional). } \\
\text { Dirigir la observación, y posibilitar la } \\
\text { interpretación de datos. }\end{array}$ & $\begin{array}{l}\text { En F.D.: consideraciones de mayor } \\
\text { generalidad que en F.I.; estos últimos } \\
\text { relacionan más fuertemente con trabajos } \\
\text { de laboratorio. }\end{array}$ \\
\hline $\begin{array}{l}\text { Empleo de } \\
\text { modelos } \\
\text { alternativos }\end{array}$ & $\begin{array}{l}\text { Es posible que en la Ciencia se acepten } \\
\text { varios modelos para un mismo sistema } \\
\text { real; pero en el marco de un cierto } \\
\text { trabajo, sólo uno es válido. } \\
\text { (Otras respuestas: dubitativas) }\end{array}$ & $\begin{array}{l}\text { Es muy frecuente que haya más de un modelo } \\
\text { que sea posible emplear. Se tiende a usar el } \\
\text { más sencillo, para tener menor complejidad en } \\
\text { las ecuaciones. }\end{array}$ & $\begin{array}{l}\text { Comentarios de F.D.: algunos dudosos, y } \\
\text { otro con referencia a la Ciencia en su } \\
\text { conjunto. } \\
\text { Los F.I. valoran la funcionalidad del } \\
\text { modelo para resolver problemas } \\
\text { experimentales }\end{array}$ \\
\hline $\begin{array}{l}\text { Posibles } \\
\text { modificaciones } \\
\text { sobre los } \\
\text { modelos en uso }\end{array}$ & $\begin{array}{l}\text { Realizadas para mejorar ajuste a la } \\
\text { teoría. } \\
\text { Para "acercar" el modelo al sistema real. }\end{array}$ & $\begin{array}{l}\text { Los modelos cambian (declaraciones } \\
\text { enfáticas). } \\
\text { Las modificaciones se realizan para mejorar el } \\
\text { ajuste del modelo a los resultados } \\
\text { experimentales. }\end{array}$ & $\begin{array}{l}\text { Para los F.D. los cambios en un modelo se } \\
\text { rigen por la estructura de la Ciencia, o bien } \\
\text { por la realidad física. } \\
\text { Para los F.I. el contexto de tales cambios } \\
\text { es el de un problema concreto de } \\
\text { laboratorio. }\end{array}$ \\
\hline
\end{tabular}

Categoría 4: Empleo de modelos en las clases universitarias

\begin{tabular}{||l|l|l|l||}
\hline & \multicolumn{1}{|c|}{ FUTUROS DOCENTES (F.D.) } & \multicolumn{1}{|c|}{ FUTUROS INVESTIGADORES (F.I.) } & \multicolumn{1}{c|}{ COMENTARIOS } \\
\hline $\begin{array}{l}\text { Modelos en toda la } \\
\text { Fisica }\end{array}$ & Expresiones dubitativas. & $\begin{array}{l}\text { La importancia del modelado no depende de la } \\
\text { temática en estudio. }\end{array}$ & $\begin{array}{l}\text { Ambos grupos están de acuerdo, pero } \\
\text { es más firme entre los F.I. }\end{array}$ \\
\hline $\begin{array}{l}\text { Cuando eran novatos } \\
\text { no advertían el uso } \\
\text { de modelos }\end{array}$ & $\begin{array}{l}\text { Tal vez, algunos problemas de } \\
\text { comprensión se hubieran evitado si } \\
\text { tomaban conciencia de la presencia de } \\
\text { modelos }\end{array}$ & $\begin{array}{l}\text { Analizar el modelado requiere especial } \\
\text { preparación, que sólo se logra cuando se está } \\
\text { avanzado en la carrera universitaria. }\end{array}$ & $\begin{array}{l}\text { Opiniones diferentes respecto de la } \\
\text { dilación en la presentación explícita de } \\
\text { modelos }\end{array}$ \\
\hline $\begin{array}{l}\text { Ejemplos de temas } \\
\text { en los que usan } \\
\text { modelos }\end{array}$ & $\begin{array}{l}\text { Ejemplos prototípicos. Capítulos de la } \\
\text { Física y/o asignaturas de la carrera. }\end{array}$ & $\begin{array}{l}\text { Complementan con ejemplos específicos de su } \\
\text { tarea de investigación. }\end{array}$ & $\begin{array}{l}\text { Los F.I. dan mayor variedad de } \\
\text { ejemplos. }\end{array}$ \\
\hline \hline
\end{tabular}

la comprensión, en tanto que los F.I. acuerdan en que las reflexiones sobre el modelado no han de plantearse hasta que el estudiante esté adecuadamente preparado para ello.
El tipo de ejemplificaciones que ofrecen sobre los modelos que recuerdan utilizar es más rico y variado entre los F.I. que en los F.D. Se advierte en los primeros la influencia de la realización de trabajos de investigación. 
Finalmente, cabe llamar la atención sobre algunos rasgos del discurso que marcan, también, diferencias entre uno y otro grupo de estudiantes. Algunos de estos rasgos pueden apreciarse en los fragmentos transcriptos en este informe y son, por ejemplo: la extensión de los turnos de habla de los entrevistados, que es mayor en el caso de los F.I. que entre los F.D. advirtiéndose en los primeros, además, mayor seguridad en sus argumentaciones, con menor frecuencia de expresiones dubitativas; algo similar ocurre con el nivel de profundidad con que se analizan los diversos tópicos. Estos rasgos pueden considerarse indicadores de diferencias en cuanto a la asiduidad con la que los estudiantes han tenido oportunidad de emplear modelos y de analizar su relación con la realidad, así como de conocer "desde adentro" algunas de las tareas de modelado que se realizan en la comunidad científica.

\section{Comentarios finales}

Entre los estudiantes entrevistados en este trabajo se encuentran diferencias en sus concepciones de modelo científico que son atribuibles a su experiencia en tareas de investigación en Física. El contacto de los F.D. con investigadores está circunscripto a la relación docente/alumno y tal como consignáramos en otro trabajo (Islas-Pesa, 2000 a) las cuestiones referidas al modelado sólo están presentes de modo explícito en las cátedras universitarias de los últimos aos de la carrera, razón por la cual estos estudiantes (que no realizan investigación) tienen dificultades para reconocer la presencia permanente de los modelos en todo estudio físico.

Para la población estudiada en esta investigación exploratoria se encuentra una distancia entre las concepciones de modelo de uno y otro segmento poblacional que alerta sobre la importancia de mejorar la formación docente en este aspecto, ya que puede suponerse que estos F.D. necesitan mejorar su competencia para manejarse en estas cuestiones. En otro trabajo (Islas-Pesa, 2000 b) realizado con docentes en ejercicio encontramos dificultades similares a las que aquíse han detallado para los F.D. En ambos casos, los resultados sugieren que la superación de estas dificultades puede abordarse intensificando la vinculación de los docentes con los ámbitos de producción del conocimiento, y generando espacios para la reflexión sobre temas de epistemología.

\section{Bibliografia}

L. LAUDAN, El progreso y sus problemas. Ed. Encuentro. España. (1986).

M. BUNGE. La investigación científica. Ed. Ariel, España (1985).

N. NERSESSIAN, Should Physicists Preach What They Practice?. Science \& Education, 4, 203 (1995).
J. SNYDER, An investigation of the knowledge structures of experts, intermediates and novices in physics. International Journal of Science Education, 22, 979 (2000).

G. BACHELARD,La formación del espíritu científico. Siglo XXI Editores, México. (1991).

H. MEYLING, How to Change Students' Conceptions of the Epistemology of Science. Science \& Education, 6, 397 (1997).

J. RYDER, J. LEACH, R. DRIVER, Undergraduate Science Students' Images of Science. Journal of Research in Science Teaching, 36, 201 (1999).

S. TOULMIN La comprensión humana. Tomo 1: El uso colectivo y la evolución de conceptos. Alianza Editorial. Madrid (1977).

L. GROSSLIGHT, C. UNGER, E. JAY, Understanding Models and their Use in Science: Conceptions of Middle and High School Students and Experts. Journal of Research in Science Teaching, 28, 799 (1991).

S. ISLAS , M. PESA, El manejo de modelos científicos en las clases universitarias. Memorias del V Simposio de Investigadores en Educación en Física. Santa Fe, Argentina (2000 a).

A. HARRISON, D. TREAGUST, A typology of school science models. International Journal of Science Education, 22, 1011 (2000).

L. CUDMANI, J. SALINAS, M. PESA, Modelo y realidad - Taller desarrollado durante la VII Reunión Nacional de Educación en Física. Mendoza, Argentina. (1991).

J. GILBERT, C. BOULTER, M. RUTHERFORD, Models in explanations, Part 1: Horses for courses?. International Journal of Science Education, 20, 83 (1998). J. GOBERT, B. BUCKLEY, Introduction to modelbased teaching and learning in science education. International Journal of Science Education, 22, 891 (2000). S. ISLAS, M. PESA, La resignificación de los modelos científicos en las aulas: ideas de docentes sobre el modelado en Física. Memorias del II Congreso Iberoamericano de Educación en Ciencias Experimentales. Córdoba, Argentina. (2000 b).

R. JUSTI, J. GILBERT, History and philosophy of science trough models: some challenges in the case of atom. International Journal of Science Education, 22, 993 (2000).

S. W. GILBERT, Model building and a definition of Science. Journal of Research in Science Teaching, 28, 73 (1991).

A. HARRISON, D. TREAGUST, Secondary Students' Mental Models of Atoms and Molecules: Implications for Teaching Chemistry. Science Education, 80, 509 (1996).

I. HALLOUN, Schematic Modeling for Meaninful Learning of Physics. Journal of Research in Science Teaching, 33, 1019 (1996).

M. L. AIELLO-NICOSIA, R. M. SPERANDEOMINEO, Educational reconstruction of physics content 
to be taught of pre-service teacher training: a case study. International Journal of Science Education, 22, 1085 (2000).

J. SAMAJA, Epistemología y metodología. Eudeba. Buenos Aires. (1993) W. FIRESTONE, Alternative Arguments for Generalizing From Data as Applied to Qualitative Research. Educational Researcher, 22, 16 (1993).

I. DEY, Qualitative Data Analysis. Ed. Rotledge. USA. (1993).

\section{ANEXO 1 \\ Protocolo de entrevistas a estudiantes universitarios $^{3}$}

1) ¿Qué ideas vienen a su mente al escuchar la palabra "modelo" en Física?
2) ¿Considera que en las clases es frecuente el uso de modelos? ¿Tiene la misma importancia en las diferentes asignaturas de la carrera universitaria?

3) ¿Puede mencionar ejemplos de modelos que se emplean con frecuencia en las clases universitarias?

4) Como estudiante universitario, ¿puede identificar cuáles son los aspectos que le resultan más "difíciles" en el manejo de modelos?

5) ¿Recuerda situaciones de clase en la cual se haya tratado el tema de la elaboración y empleo de modelos? (no, de un modelo en particular, sino de los modelos, en general).

6) En su opinión, ¿para qué se elaboran los modelos? ¿Cuál es el propósito de los modelos?

7) ¿Cuáles son las características que Ud. conoce (o supone) en cuanto al uso que hacen los científicos de los modelos? ¿Es frecuente que un científico cambie de modelo? ¿Puede un científico tener más de un modelo para resolver una misma situación problemática?

8) ¿Cuál sería la relación entre modelo y realidad? ¿Cuán cerca debe estar un modelo de las cosas en sí mismas?

\footnotetext{
${ }^{3}$ Algunas de estas preguntas fueron extraídas de Grosslight et al. (1991)
} 